\title{
A High-Accurate Fast Poisson Solver Based on Harmonic Surface Mapping Algorithm
}

\author{
Jiuyang Liang ${ }^{1}$, Pei $\mathrm{Liu}^{2}$ and Zhenli $\mathrm{Xu}^{1, *}$ \\ 1 School of Mathematical Sciences, Institute of Natural Sciences, and MOE-LSC, \\ Shanghai Jiao Tong University, Shanghai 200240, China. \\ 2 School of Mathematics, University of Minnesota, Twin Cities, Minneapolis, \\ MN 55455, USA.
}

Received 21 March 2020; Accepted (in revised version) 20 November 2020

\begin{abstract}
Poisson's equations in a cuboid are frequently solved in many scientific and engineering applications such as electric structure calculations, molecular dynamics simulations and computational astrophysics. In this paper, a fast and highly accurate algorithm is presented for the solution of the Poisson's equation in a cuboidal domain with boundary conditions of mixed type. This so-called harmonic surface mapping algorithm is a meshless algorithm which can achieve a desired order of accuracy by evaluating a body convolution of the source and the free-space Green's function within a sphere containing the cuboid, and another surface integration over the spherical surface. Numerical quadratures are introduced to approximate the integrals, resulting in the solution represented by a summation of point sources in free space, which can be accelerated by means of the fast multipole algorithm. The complexity of the algorithm is linear to the number of quadrature points, and the convergence rate can be arbitrarily high even when the source term is a piecewise continuous function.
\end{abstract}

AMS subject classifications: 35J08, 35Q70, 33F05, 78M16

Key words: Fast algorithm, Poisson's equation, boundary integral method, image charge, mixed boundary condition, fast multipole method.

\section{Introduction}

The solution of Poisson's equation plays an essential role in scientific computing as well as many physical and engineering applications such as molecular simulations, electric structure calculations, computational astrophysics, and fluid dynamics for both particle simulations [1-4] and continuum-theory calculations [5-8]. The development of efficient

*Corresponding author. Email addresses: liangjiuyang@sjtu.edu.cn (J. Liang), liu01304@umn.edu (P. Liu), xuzl@sjtu.edu.cn (Z. Xu) 
method for the Poisson's equation in these fields remains an important theme for simulations using high-performance computing. A great deal of numerical approaches have been developed, subject to different geometry and boundary conditions such as gridbased methods based on finite element or finite difference discretizations [9]. To reduce the number of grid elements, the boundary integral/element method [10-12] reformulates the Poisson's equation into a Fredholm integral equation of the second kind and can be accelerated through the fast multipole method (FMM) [13, 14].

Despite many advances on complex domains, fast numerical solvers for Poisson's equation of constant coefficient in a cuboidal domain remain to be an important theme due to its significance in many applications. Various numerical approaches especially for large scale parallel solvers have been proposed for this problem which are extensively used in practice. Most of the algorithms have second order of accuracy and $\mathcal{O}(n \log n)$ computational complexity, with $n$ being the total number of grid points, such as the finite difference method with fast Fourier transform (FFT) or geometric multigrid, and the finite element method with proper preconditioning strategy [15]. Higher order schemes are also available by using high-order elements. When the source term and boundary conditions are sufficiently smooth, the spectral method based on orthogonal polynomials could converge exponentially while the computation cost remains to be expensive, and efficient implementations can achieve $\mathcal{O}\left(n^{3 / 2}\right)$ for two dimensions and $\mathcal{O}\left(n^{4 / 3}\right)$ for three dimensions using Legendre or Chebyshev polynomials [16,17]. For certain type of boundary conditions, the pseudospectral Fourier method with polynomial subtraction technique to eliminate the Gibbs phenomenon can also achieve high order accuracy [18,19]. For specific boundary conditions in a regular domain, the method of image can be used to represent the solution into an infinite sum or integral over the whole 3D space [20,21]. The solution of Poisson's equation in the whole 3D space can be written as the convolution of the free-space Green's function and the source term. This convolution can be efficiently evaluated with high order accuracy through the FMM-based methods [20,22], the method of local corrections [23,24], or the FFT-based methods [25,26].

Recently, a harmonic surface mapping algorithm (HSMA), which combines the method of image charges and the boundary integral method to calculate the Green's function, has been proposed and applied to particle systems $[27,28]$. Its basic idea is that an auxiliary surface is introduced such that the contribution of image charges outside the surface is represented by image charges distributed over the surface. The harmonic surface mapping is a procedure to transform the local expansion from the exterior contribution into the surface integral which can be discretized into a sum of image charges. This algorithm is efficient to calculate the infinite sum for particle interaction in a box with various boundary conditions, especially non-periodic conditions when the FFT-based lattice summation cannot be directly applied.

The algorithm developed in this work is the extension of the HSMA, aiming to solve the Poisson's equation in a rectangular domain with mixed-type boundary conditions, which also introduces the auxiliary surface and uses a surface integral to represent the integral outside the surface. The main difficulty of this extension is the treatment of sin- 
gularity in the integral, particularly, when a singular point is near the domain of less smoothness. We introduce the Laplace asymptotic method to analytically treat the singularity contribution, which significantly improves the computational efficiency. The asymptotic error can be controlled by a parameter such that the error can be smaller than a given tolerance. In addition, we improve the surface mapping result of Zhao et al. [27] where the surface dipole derived by the interior boundary integral expression is replaced by a surface charge by the exterior boundary integral, which reduces the complexity in approximating the boundary integral. The computational complexity of the algorithm is linear to the quadrature points through the FMM acceleration. The order of convergence rate could be arbitrary even with piecewise continuous source terms. Considering the low computational cost and high convergence rate, the new HSMA for the Poisson equation is very promising for practical applications.

The organization of this paper is as follows. In Section 2, we discuss the integral expression of the Poisson's equation by the continuation with the image charge method (ICM). In Section 3, we present the HSMA formulation and numerical approximation. In Section 4, numerical examples are given to show the attractive performance of the algorithm. Concluding remarks are given in Section 5.

\section{Integral expression of the Poisson's equation}

In this paper, we consider the solution of the following Poisson's equation in a cuboidal domain $\Omega=\left[-L_{x} / 2, L_{x} / 2\right] \times\left[-L_{y} / 2, L_{y} / 2\right] \times\left[-L_{z} / 2, L_{z} / 2\right]$ in three dimensions,

$$
-\nabla^{2} u(\boldsymbol{r})=f(\boldsymbol{r}), \quad \text { in } \Omega,
$$

subject to some boundary conditions $\mathcal{L} u=0$ on $\partial \Omega$. For convenience of description, one supposes that the source term $f(\boldsymbol{r})$ is piecewise smooth in $\Omega$. The case of $f(\mathbf{r})$ being the sum of delta functions corresponds to a point-charge distribution, for which the solution can also be accurately calculated as described in our prior work [27]. The boundary condition on each face of $\partial \Omega$ can take either Dirichlet type $u=0$, Neumann type $\partial u / \partial n=$ 0 , periodic, dielectric or free-space boundary condition. Nonhomogeneous boundary conditions can be directly taken into account through the surface integrals from potential theory and will not be discussed here.

The goal of this paper is fast evaluation of the convolution expression of the solution with the help of the ICM. The ICM is a class of techniques aiming at obtaining the Green's function in the presence of boundary conditions [29]. It benefits from the regular shape of the boundary so that the boundary condition can be inferred by placing a small number of fictitious charges of certain valences at some locations outside the volume $\Omega$. The fictitious charges are called image charges. For a cuboidal geometry, most boundary conditions can be handled by the ICM with a series of image charges through the reflections between boundaries. The most intuitive case is the periodic boundary condition for which the image charges are simply the duplication of the source point in each periodic 
copy of the central box. For other geometries, the image charges can also be obtained by transforming from the harmonic series [30] or by the least-square fitting [31].

For boundary conditions other than the periodicity, the ICM can be constructed by first solving the problem with one planar boundary and then obtaining a series of images by reflections between boundaries using those one-plane formulas. Consider the Green's function problem, $-\nabla^{2} G\left(\boldsymbol{r}, \boldsymbol{r}^{\prime}\right)=\delta\left(\boldsymbol{r}-\boldsymbol{r}^{\prime}\right)$, in the half space $x>0$, with $\boldsymbol{r}=(x, y, z), \boldsymbol{r}^{\prime}=$ $\left(x^{\prime}, y^{\prime}, z^{\prime}\right)$ and $x^{\prime}>0$. Let $r^{\prime \prime}=\left(-x^{\prime}, y^{\prime}, z^{\prime}\right)$ be the point of mirror symmetry and the image charge strength at this point is $Q$, and thus the solution of the Green's function is the electric potential by the superposition of the point source at $r^{\prime}$ and the point image at $\boldsymbol{r}^{\prime \prime}$, that is, $G\left(\boldsymbol{r}, \boldsymbol{r}^{\prime}\right)=\left(1 /\left|\boldsymbol{r}-\boldsymbol{r}^{\prime}\right|+Q /\left|\boldsymbol{r}-\boldsymbol{r}^{\prime \prime}\right|\right) / 4 \pi$. Depending on the boundary condition specified at $x=0$, the strength $Q$ can be expressed as:

(I) For Dirichlet $\mathrm{BC}, \mathrm{G}=0$ at $x=0$, the image strength is $Q=-1$;

(II) For Neumann BC, $\partial_{x} G=0$ at $x=0$, the image strength is $Q=1$;

(III) For dielectric BC such that the electric potential $G$ and the electric displacement $\varepsilon \partial_{x} G$ are continuous across the interface at $x=0$, where $\varepsilon$ is the dielectric permittivity which equals one for $x>0$. Let the dielectric coefficient for $x<0$ is $\epsilon^{\prime}$. Then the image strength is $Q=\left(1-\epsilon^{\prime}\right) /\left(1+\epsilon^{\prime}\right)$.

Now we proceed to derive the continuation of the source in the whole space $\mathbb{R}^{3}$. We divide the space into cells of the same size as the central box $\Omega$ such that $\Omega_{i j k}$ is the cell centered at $\left(i L_{x}, j L_{y}, k L_{z}\right)$ with the indices $i, j$ and $k$, and $\Omega_{000}=\Omega$ (see Fig. 1). Let $g_{i j k}(\boldsymbol{r})$ with $r \in \Omega_{i j k}$ become the representation of the continuation function of $f(r)$ in cell $\Omega_{i j k}$ where $g_{000}(\boldsymbol{r})=f(\boldsymbol{r})$, we obtain $g_{i j k}$ through the following procedure.

Due to the boundary condition on each of the six boundaries, the continuation in a cell contacting with the central box can be performed to satisfy the boundary condition at the shared boundary. For example, $g_{100}$ is the image source of $g_{000}$ due to the boundary condition at $x=L_{x} / 2$. By the one-plane ICM, the image source for a continuous distribution,

$$
g_{100}(x, y, z)=Q g_{000}\left(L_{x}-x, y, z\right) .
$$

For a cell not contacting with the central box, the image source can be constructed by the reflection between boundaries. A cell of index ijk can be considered as the image of the source in the cell of index $\tilde{i} j k$ due to the boundary condition at $x=\operatorname{sign}(i) L_{x} / 2$, where $\tilde{i}=-\operatorname{sign}(i)(|i|-1)$, namely,

$$
g_{i j k}(x, y, z)=Q g_{\tilde{i j k}}\left(\operatorname{sign}(i) L_{x}-x, y, z\right) .
$$

For the continuation along the $y$ and $z$ directions, it can be also obtained by the image reflection using the one-plane ICM due to the $y$ or $z$ boundaries, and the constructed image source will be the same as Eq. (2.3). One then reformulates the solution $u(r)$ where 


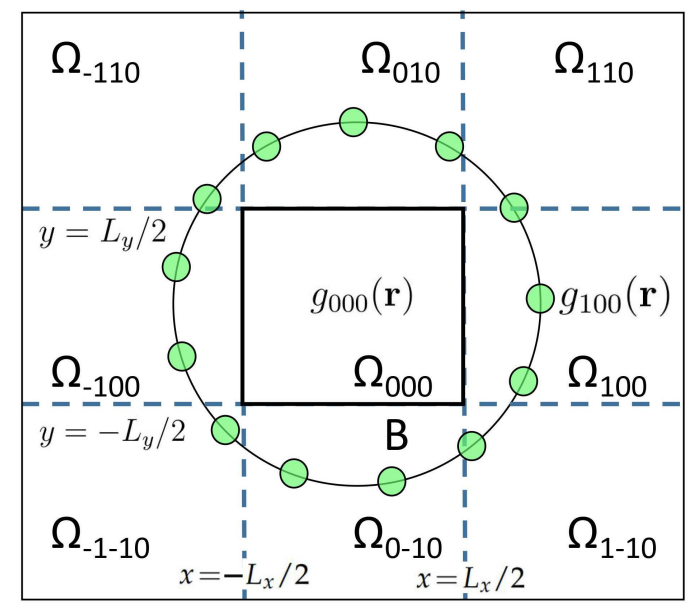

Figure 1: Schematic (2D illustration for 3D problem) of the ICM and the HSMA algorithm in a cuboidal domain. ICM transforms the boundary-value problem into a volume integral over 3D space. The contribution outside $B$ will be represented by surface integral on $\partial B$.

$r \in \Omega$ into an integral,

$$
\begin{aligned}
u(\boldsymbol{r}) & =\sum_{i j k} \int_{\Omega_{i j k}} g_{i j k}\left(\mathbf{r}^{\prime}\right) G_{0}\left(\mathbf{r}, \mathbf{r}^{\prime}\right) d \mathbf{r}^{\prime} \\
& =\int_{\mathbb{R}^{3}} g\left(\boldsymbol{r}^{\prime}\right) G_{0}\left(\boldsymbol{r}, \boldsymbol{r}^{\prime}\right) d \boldsymbol{r}^{\prime},
\end{aligned}
$$

where $G_{0}\left(\boldsymbol{r}, \boldsymbol{r}^{\prime}\right)=1 /\left(4 \pi\left|\boldsymbol{r}-\boldsymbol{r}^{\prime}\right|\right)$ is the fundamental solution of the Poisson's equation. $g\left(\boldsymbol{r}^{\prime}\right)=\sum_{i j k} \chi_{i j k}\left(\boldsymbol{r}^{\prime}\right) g_{i j k}\left(\boldsymbol{r}^{\prime}\right)$, with $\chi_{i j k}$ denoting the indicative function of $\Omega_{i j k}$, is the continuation function of $f(\boldsymbol{r})$ in $\mathbb{R}^{3}$ such that the solution $u$ satisfies the specified boundary condition. The determination of $u$ will be discussed in the following subsection.

It is straightforward to see that function $u(r)$ given by (2.4) satisfies Eq. (2.1) in $\Omega$ as well as the boundary conditions on $\partial \Omega$. Now the problem becomes how to numerically evaluate the above integral. In the literature, there are extensive efforts $[1,21,32-35]$ in developing fast algorithms for periodic sums based on the periodic structure of the source term. Here we adapt the HSMA algorithm [27] to construct an efficient and accurate method for general boundary conditions.

\section{Harmonic surface mapping algorithm}

\subsection{Local expansion of the exterior integral}

In order to efficiently calculate the infinite integral (2.4), we introduce a larger ball $B$ of radius $R$ with the center at the origin such that $\Omega \subset B$. The integral can be split into two 
components,

$$
u(\boldsymbol{r})=\int_{B} g\left(\boldsymbol{r}^{\prime}\right) G_{0}\left(\boldsymbol{r}, \boldsymbol{r}^{\prime}\right) d \boldsymbol{r}^{\prime}+\int_{B^{c}} g\left(\boldsymbol{r}^{\prime}\right) G_{0}\left(\boldsymbol{r}, \boldsymbol{r}^{\prime}\right) d \boldsymbol{r}^{\prime} \triangleq V(\boldsymbol{r})+W(\boldsymbol{r}),
$$

where $V(\boldsymbol{r})$ and $W(\boldsymbol{r})$ represent the integrals inside and outside the ball, respectively. Since $W(\boldsymbol{r})$ is a harmonic function in $B$, it can be approximated by the spherical harmonic expansion in spherical coordinate $r=(r, \theta, \psi)[21,27]$, truncated at $n=P$,

$$
W(r, \theta, \psi) \approx \sum_{n=0}^{P} \sum_{m=-n}^{n} A_{n}^{m} r^{n} Y_{n}^{m}(\theta, \psi),
$$

where $Y_{n}^{m}$ is the spherical harmonic function of degree $n$ and order $m$. Here, $\left\{A_{n}^{m}, n=\right.$ $0, \cdots, P, m=-n, \cdots, n\}$ is a set of unknown coefficients. In principle, a small amount of spherical harmonics would be enough to the desired accuracy thanks to the spectral convergence of the series, which means the total number of unknown coefficients, $N_{b}=$ $(P+1)^{2}$, can be small. These coefficients will be determined by a linear-square fitting. Then the numerical solution $u(r)$ as well as its derivatives are computed based on,

$$
u(\boldsymbol{r})=V(\boldsymbol{r})+\sum_{n=0}^{P} \sum_{m=-n}^{n} A_{n}^{m} r^{n} Y_{n}^{m}(\theta, \psi)
$$

The evaluation of $V(r)$ can be obtained by volume integral scheme and the details will be given later on. To obtain coefficients $A_{n}^{m}$, we first generate $N_{m}$ monitoring points $\left\{\boldsymbol{r}_{i}=\left(r_{i}, \theta_{i}, \psi_{i}\right), i=1, \cdots, N_{m}\right\}$ on the boundary $\partial \Omega$. We take $N_{m}>N_{b}$. Then coefficients $A_{n}^{m}$ are obtained by minimizing the residual norm of the boundary conditions at these points [27],

$$
\left\{A_{n}^{m}\right\}=\operatorname{argmin} \sum_{i=1}^{N_{m}}\left|\mathcal{L}\left(u\left(\boldsymbol{r}_{i}\right)\right)\right|^{2}=\operatorname{argmin}\|M A-Y\|_{L^{2}} .
$$

Notice that $\mathcal{L}$ is linear in $A_{n}^{m}$, and it can be expressed using an $N_{m}$-by- $N_{b}$ matrix $M$ and a vector $Y$. Here $A$ represents the vector consisting all $A_{n}^{m}$. Matrix $M$ depends on locations of monitoring points and the order and degree of the harmonic series and can be constructed in the preparation step. One can perform $Q R$ factorization to matrix $M$ to solve the least-square problem.

It is noted that periodic boundary conditions are different from other boundary conditions because the distribution of monitoring points on $\partial \Omega$ will lead to an ill-conditioned matrix [21]. To avoid that, one should select the monitoring points $\left\{\boldsymbol{r}_{i}\right\}$ on the circumsphere of the cuboidal domain $\Omega$. Let $\widetilde{\boldsymbol{r}}_{i}=\boldsymbol{r}_{i}-\boldsymbol{k} \boldsymbol{L}$ with $\boldsymbol{L}=\left(L_{x}, L_{y}, L_{z}\right)$ and $\boldsymbol{k}$ being the index vector such that $\widetilde{r}_{i}$ is located within the central domain $\Omega$. The $i$ th component of the vector $Y$ is then given by $Y_{i}=V\left(\boldsymbol{r}_{i}\right)-V\left(\widetilde{\boldsymbol{r}}_{i}\right)$, which leads to a better conditioned fitting matrix $M$. The condition number can be further reduced by an appropriate distribution of these monitoring points. One suggestion is the distribution which is obtained by solving the Thompson problem of the equilibrium position of mutually repelling electrons 
constrained to be on the surface of the sphere $[36,37]$. The random uniformly distributed points and the Gauss nodes [38] can also be used as the suggested distribution. The comparative results can be found in [21,27]. In this work, we use the Fibonacci grid [39], which arranges the monitoring points along a spiral lattice such that they are close to a uniform distribution on the spherical surface.

\subsection{Surface mapping from the Green's identity}

The key idea of the HSMA is the map from the series of the spherical harmonic expansion of $W$ onto surface integrals over the sphere $B$, which speeds up the evaluation by means of the FMM. This is important because repeatedly evaluating spherical harmonic functions through their recursive relations is less efficient in practical simulations.

Using the harmonicity of potential $W(\boldsymbol{r})$ in $B$ and the Green's second identity, one has,

$$
W(\boldsymbol{r})=\int_{\partial B}\left(G_{0}\left(\boldsymbol{r}, \boldsymbol{r}^{\prime}\right) \frac{\partial W\left(\boldsymbol{r}^{\prime}\right)}{\partial \boldsymbol{n}^{\prime}}-W\left(\boldsymbol{r}^{\prime}\right) \frac{\partial G_{0}\left(\boldsymbol{r}, \boldsymbol{r}^{\prime}\right)}{\partial \boldsymbol{n}^{\prime}}\right) d S^{\prime}, \quad \boldsymbol{r} \in B .
$$

This shows that the potential can be described by the superposition of a surface charge distribution of density $\partial W\left(\boldsymbol{r}^{\prime}\right) / \partial \boldsymbol{n}^{\prime}$ and a surface dipole of density $W\left(\boldsymbol{r}^{\prime}\right)$ on $\partial B$. Alternatively, one can introduce another harmonic function $\widetilde{W}$ defined in domain $B^{c}$,

$$
\widetilde{W}(\boldsymbol{r})=\sum_{n=0}^{P} \sum_{m=-n}^{n} A_{n}^{m} R^{2 n+1} r^{-n-1} Y_{n}^{m}(\theta, \psi)
$$

Since $\nabla^{2} \widetilde{W}=0$ in $B^{c}$, by the Green's second identity again,

$$
\int_{\partial B^{c}}\left[G_{0}\left(\boldsymbol{r}, \boldsymbol{r}^{\prime}\right) \frac{\partial \widetilde{W}\left(\boldsymbol{r}^{\prime}\right)}{\partial \boldsymbol{n}^{\prime}}-\widetilde{W}\left(\boldsymbol{r}^{\prime}\right) \frac{\partial G_{0}\left(\boldsymbol{r}, \boldsymbol{r}^{\prime}\right)}{\partial \boldsymbol{n}^{\prime}}\right] d S^{\prime}=0,
$$

which means the double-layer potential in (3.5) can be rewritten into a single layer potential with surface charge density $\partial \widetilde{W}(\boldsymbol{r}) / \partial n$. Since $W$ and $\widetilde{W}$ are equal on the boundary $\partial B$, one has,

$$
W(\boldsymbol{r})=\int_{\partial B} G_{0}\left(\boldsymbol{r}, \boldsymbol{r}^{\prime}\right) \sigma\left(\boldsymbol{r}^{\prime}\right) d S^{\prime}, \quad \boldsymbol{r} \in B,
$$

where $\sigma\left(\boldsymbol{r}^{\prime}\right)=\partial\left(W\left(\boldsymbol{r}^{\prime}\right)-\widetilde{W}\left(\boldsymbol{r}^{\prime}\right)\right) / \partial \boldsymbol{n}^{\prime}$ is the surface charge density.

There is clearly no singularity in the surface integral (3.8). One can apply the Fibonacci numerical integration [39] to achieve a convergence rate of $N_{o}^{-6}$ with $N_{o}$ being the number of grid points. It is remarked that, in the original HSMA for particle systems [27], the surface dipole in Eq. (3.5) is discretized as two surface charges which are close to each other. In this work, we improve the result and use the formula (3.8) which avoids the approximation of the surface dipole and improves the efficiency. 


\subsection{Evaluation of the volume integral}

In this section, we discuss the numerical method for the approximation of the volume integral $V$ in Eq. (3.1), which can be rewritten as

$$
V(\boldsymbol{r})=\sum_{i j k} \int_{\Omega_{i j k} \cap B} g\left(\boldsymbol{r}^{\prime}\right) G_{0}\left(\boldsymbol{r}, \boldsymbol{r}^{\prime}\right) d \boldsymbol{r}^{\prime} \triangleq \sum_{i j k} v_{i j k}(\boldsymbol{r}) .
$$

Due to the non-smoothness of the source term $g(\boldsymbol{r})$, FFT method converges slowly. We will employ piecewise Gauss quadrature to evaluate $v_{i j k}$ considering that $g(\boldsymbol{r})$ is a piecewise smooth function. Since the geometry of $\Omega_{i j k} \cap B$ is defined by the ball and the cuboidal box, we can write the volume integral $v_{i j k}$ into an iterated integral,

$$
v_{i j k}(\boldsymbol{r})=\int_{z_{1}}^{z_{2}} \int_{g_{1}(z)}^{g_{2}(z)} \int_{h_{1}(y, z)}^{h_{2}(y, z)} g(x, y, z) G_{0}\left(\boldsymbol{r}, \boldsymbol{r}^{\prime}\right) d x d y d z,
$$

where the function $g_{1}(z), g_{2}(z), h_{1}(y, z), h_{2}(y, z)$ and the integrand $g(x, y, z)$ are all piecewise smooth. For each of the intervals such that these functions are smooth, the Gauss quadrature is correspondingly employed.

Since $G_{0}\left(\boldsymbol{r}, \boldsymbol{r}^{\prime}\right)$ is singular when $\boldsymbol{r}^{\prime} \rightarrow \boldsymbol{r}$, we first need to treat the singularity to achieve high accuracy. For $\Omega_{i j k}$ with $|i|+|j|+|k|>2$, it is away from the central box $\Omega$, thus no additional operation is required to evaluate $v_{i j k}(\boldsymbol{r})$. If $\Omega_{i j k}$ shares a corner vertex (or an edge, or a boundary face) with $\Omega$, the evaluation of $v_{i j k}(\boldsymbol{r})$ for the location close to the corner point (or the edge, or the face) might converge slowly. We discuss the techniques for the treatment to these four situations, separately.

Case 1. When $r \in \Omega_{000}$, and far from the boundaries, the Taylor expansion of the source function at $r$ can be subtracted to remove the singularity,

$$
\begin{aligned}
V(\boldsymbol{r}) & =\int_{B} \frac{g\left(\boldsymbol{r}^{\prime}\right)-T\left(\boldsymbol{r}, \boldsymbol{r}^{\prime}\right) e^{-a\left|\boldsymbol{r}^{\prime}-\boldsymbol{r}\right|^{K+1}}}{4 \pi\left|\boldsymbol{r}-\boldsymbol{r}^{\prime}\right|} d \boldsymbol{r}^{\prime}+\int_{B} \frac{T\left(\boldsymbol{r}, \boldsymbol{r}^{\prime}\right) e^{-a\left|\boldsymbol{r}^{\prime}-\boldsymbol{r}\right|^{K+1}}}{4 \pi\left|\boldsymbol{r}-\boldsymbol{r}^{\prime}\right|} d \boldsymbol{r}^{\prime} \\
& \triangleq I_{1}(\boldsymbol{r})+I_{2}(\boldsymbol{r}),
\end{aligned}
$$

where $T=\sum_{i=0}^{K} \frac{1}{i !}\left[\left(\boldsymbol{r}^{\prime}-\boldsymbol{r}\right) \cdot \nabla\right]^{i} g(\boldsymbol{r})$ is the truncated Taylor expansion of function $g$, the factor $e^{-a\left|r^{\prime}-r\right|^{K+1}}$ is introduced to localize the expansion and the parameter $a$ is a positive number. Then the integrand of $I_{1}$ is $C^{K-1}$ at $\boldsymbol{r}^{\prime} \rightarrow \boldsymbol{r}$ and piecewise $C^{\infty}$ elsewhere. The convergence rate of the $N$-point Gauss quadrature is $\mathcal{O}\left(N^{-K-3}\right)$ which can be proved by extending the asymptotic error estimate [40] to three dimensions.

The integral $I_{2}$ can be approximated by shifting the integral variable $r^{\prime}$ to $r^{\prime \prime}=r^{\prime}-r$ defined on $\mathbb{R}^{3}$,

$$
I_{2}=\sum_{i=0}^{K} \frac{1}{i !} \iiint_{\mathbb{R}^{3}} \frac{\left[\boldsymbol{r}^{\prime \prime} \cdot \nabla\right]^{i} g(\boldsymbol{r}) e^{-a\left(r^{\prime \prime}\right)^{K+1}}}{4 \pi r^{\prime \prime}} d \boldsymbol{r}^{\prime \prime}+\mathcal{O}\left(e^{-a D^{K+1}}\right) .
$$


Each integral can be evaluated analytically by the Laplace asymptotic method [41]. For example, if we choose $K=3$, the leading term is,

$$
I_{2} \approx \pi g(\boldsymbol{r}) \frac{\Gamma\left(\frac{1}{2}\right)}{\sqrt{a}}+\frac{\pi \nabla^{2} g(\boldsymbol{r})}{6 a} .
$$

By choosing a relatively large $a$, the error in $I_{2}$ could be very small. However, a large $a$ will result in the integrand in $I_{1}$ to be relatively sharp and require more quadrature points to properly capture its shape. In reality, we need to balance these considerations.

Case 2. The prescribed method works poorly if $\boldsymbol{r}=\left(x_{0}, y_{0}, z_{0}\right)$ is close to a surface of $\Omega_{000}$. For example, when $x$ is close to $L_{x} / 2$, the integrand of $I_{1}$ in $\Omega_{100}$ would be sharp although not singular, which makes the convergence rate of the Gauss quadrature to be slow. To determine whether $r$ is close to $x=L_{x} / 2$ or not, one compares $\exp \left(-a\left|\mathbf{r}-\mathbf{r}_{0}\right|^{K+1}\right)$ to a small tolerance $\epsilon_{t o l}$ with $r_{0}=\left(L_{x} / 2, y, z\right)$. If it is bigger than the tolerance, the residual of the approximation given in Eq.(3.12) is not small and $r$ is considered to be close to the surface.

In this situation, we will use the Taylor expansion of $g_{000}$ and $g_{100}$ at the point $r_{0}$. The ball $B$ is divided into two parts by the plane $x=L_{x} / 2$, denoted $B^{-}$for the part $x<L_{x} / 2$ and $B^{+}$for the part $x>L_{x} / 2$. Then,

$$
\begin{aligned}
V(\boldsymbol{r})= & I_{1}+I_{2}+I_{3}+I_{4} \\
= & \int_{B^{-}} \frac{g\left(\boldsymbol{r}^{\prime}\right)-T^{-} e^{-a\left|r^{\prime}-r_{0}\right|^{K+1}}}{4 \pi\left|\boldsymbol{r}-\boldsymbol{r}^{\prime}\right|} d \boldsymbol{r}^{\prime}+\int_{B^{-}} \frac{T^{-} e^{-a\left|\boldsymbol{r}^{\prime}-\boldsymbol{r}_{0}\right|^{K+1}}}{4 \pi\left|\boldsymbol{r}-\boldsymbol{r}^{\prime}\right|} d \boldsymbol{r}^{\prime} \\
& +\int_{B^{+}} \frac{g\left(\boldsymbol{r}^{\prime}\right)-T^{+} e^{-a\left|\boldsymbol{r}^{\prime}-\boldsymbol{r}_{0}\right|^{K+1}}}{4 \pi\left|\boldsymbol{r}-\boldsymbol{r}^{\prime}\right|} d \boldsymbol{r}^{\prime}+\int_{B^{+}} \frac{T^{+} e^{-a\left|\boldsymbol{r}^{\prime}-\boldsymbol{r}_{0}\right|^{K+1}}}{4 \pi\left|\boldsymbol{r}-\boldsymbol{r}^{\prime}\right|} d \boldsymbol{r}^{\prime},
\end{aligned}
$$

where $T^{-}=\sum_{i=0}^{K} \frac{1}{i !}\left[\left(\boldsymbol{r}^{\prime}-\boldsymbol{r}_{0}\right) \cdot \nabla\right]^{i} g_{000}\left(\boldsymbol{r}_{0}\right)$ and $T^{+}=\sum_{i=0}^{K} \frac{1}{i !}\left[\left(\boldsymbol{r}^{\prime}-\boldsymbol{r}_{0}\right) \cdot \nabla\right]^{i} g_{100}\left(\boldsymbol{r}_{0}\right)$. Again the integrand in $I_{1}$ and $I_{3}$ are all $C^{K-1}$ at $\boldsymbol{r}^{\prime} \rightarrow \boldsymbol{r}$ and $C^{\infty}$ elsewhere, so that Gauss quadrature converges in the rate of $\mathcal{O}\left(N^{-K-3}\right)$.

The integral $I_{2}$ and $I_{4}$ can be approximately evaluated,

$$
\left\{\begin{array}{l}
I_{2}=\sum_{i=0}^{K} \frac{1}{i !} \iiint_{x<0} \frac{\left[\boldsymbol{r}^{\prime \prime} \cdot \nabla\right]^{i} g(\boldsymbol{r}) e^{-a\left(r^{\prime \prime}\right)^{K+1}}}{4 \pi r^{\prime \prime}} d \boldsymbol{r}^{\prime \prime}+\mathcal{O}\left(e^{-a D^{K+1}}\right), \\
I_{4}=\sum_{i=0}^{K} \frac{1}{i !} \iiint_{x>0} \frac{\left[\boldsymbol{r}^{\prime \prime} \cdot \nabla\right]^{i} g(\boldsymbol{r}) e^{-a\left(r^{\prime \prime}\right)^{K+1}}}{4 \pi r^{\prime \prime}} d \boldsymbol{r}^{\prime \prime}+\mathcal{O}\left(e^{-a D^{K+1}}\right) .
\end{array}\right.
$$

These integrals can be computed analytically.

Case 3. When $r$ is close to an edge of $\Omega_{000}$, for example $r=\left(x_{0}, y_{0}, z_{0}\right)$ close to $\left(L_{x} / 2, L_{y} / 2, z\right)$. We use the Taylor expansion of $g_{000}, g_{100}, g_{010}$ and $g_{110}$ at the point $\left(L_{x} / 2, L_{y} / 2, z\right)$. The domain $B$ is divided into four parts by the two perpendicular planes $x=L_{x} / 2$ and $y=L_{y} / 2$. Similar to Case 2, each part corresponds to a Gauss quadrature and an approximated analytical expression. 
Case 4. When $r$ is close to a corner of $\Omega_{000}$, for example $\boldsymbol{r}=\left(x_{0}, y_{0}, z_{0}\right)$ close to the corner $\left(L_{x} / 2, L_{y} / 2, L_{z} / 2\right)$. We use the Taylor expansion of all $g_{000}, g_{100}, g_{010}$ and $g_{110}, g_{001}, g_{101}$, $g_{011}$ and $g_{111}$ at the point $\left(L_{x} / 2, L_{y} / 2, L_{z} / 2\right)$. The domain $B$ is divided into eight parts by the three perpendicular planes $x=L_{x} / 2, y=L_{y} / 2$ and $z=L_{z} / 2$. Similar to Cases 2 and 3 , each part corresponds to a Gauss quadrature and an approximated analytical expression.

Remark 3.1. When evaluating $\nabla V(\boldsymbol{r})$, we can use the same method as described. The only difference is the kernel function becomes $\nabla G\left(r, r^{\prime}\right)$. The convergence rate will be 1 order lower.

Remark 3.2. To evaluate the summations in the Gauss quadrature, we use the bbFMM [42], which is a fast multipole formulation for non-oscillatory kernel with small prefactor.

\subsection{Summary of the algorithm}

In summary, the Poisson solver is composed of the following steps.

Initially, one introduces a ball $B$ of radius $R$, which includes the central box $\Omega$. The image source function $g_{i j k}(\boldsymbol{r})$ within $B$ is generated as described in Section 2, and the monitoring points are distributed on the boundary of $\Omega$. One then constructs the matrix $M$ for the harmonic surface mapping according to boundary conditions and computes the $Q R$ factorization of $M$. Since the size of $M$ is $N_{m}$-by- $N_{b}$, the QR factorization has complexity $\mathcal{O}\left(N_{m} N_{b}^{2}\right)$.

Next, one evaluates $V(\boldsymbol{r})=\int_{B} g\left(\boldsymbol{r}^{\prime}\right) G_{0}\left(\boldsymbol{r}, \boldsymbol{r}^{\prime}\right) d \boldsymbol{r}^{\prime}$ and its derivatives if any at the monitoring points according to the boundary conditions, using the method described in Section 3. The number of quadrature points $N_{s}$ is order of $N^{3}$ for the use of the $N$-point Gauss quadrature in each dimension. The complexity of this step is $\mathcal{O}\left(N_{s}+N_{m}\right)$ by using the FMM. The least-square problem is then solved to get coefficients $A_{n}^{m}$ with complexity $\mathcal{O}\left(N_{b}^{2}\right)$, and the image charges on surfaces are computed by Eq. (3.8) and Fibonacci integration with complexity $\mathcal{O}\left(N_{b} N_{o}\right)$.

Finally, for any given point $\boldsymbol{r} \in \Omega, u(\boldsymbol{r})=V(\boldsymbol{r})+W(\boldsymbol{r})$ is evaluated using the method described in Section 3 and the FMM with complexity $\mathcal{O}\left(N_{S}+N_{o}\right)$.

Remark 3.3. The total complexity of the full algorithm is $\mathcal{O}\left(N_{s}+N_{m} N_{b}^{2}+N_{b} N_{o}\right)$. In the case of $N_{m}, N_{b}$ and $N_{o}$ much smaller than $N_{s}$, the complexity is $\mathcal{O}\left(N_{s}\right)$ and the algorithm is linear scaling.

\section{Numerical examples}

The performance of our algorithm is tested by three examples. The situation with a point source has been discussed in [27]. In this section, we focus on smooth source terms (Example 4.1) and piecewise smooth source terms (Examples 4.2 and 4.3). In the calculations, we solve Poisson's equations in a cubic box $\Omega=[-1,1]^{3}$, and the reference "exact" solution uses the result of higher accuracy. The number of monitoring points is fixed to be $N_{m}=2 P^{2}$ which approximates $2 N_{b}$. 
Example 4.1. Consider the Poisson's equation $-\nabla^{2} u=f$ in the cube $\Omega$ with a smooth function source,

$$
f(\boldsymbol{r})=-2\left[\left(x^{2}-1\right)\left(y^{2}-1\right)+\left(y^{2}-1\right)\left(z^{2}-1\right)+\left(x^{2}-1\right)\left(z^{2}-1\right)\right],
$$

and Dirichlet boundary condition $\left.u\right|_{\partial \Omega}=0$, which has exact solution $u(x, y, z)=\left(x^{2}-\right.$ 1) $\left(y^{2}-1\right)\left(z^{2}-1\right)$. Four different source points are used to test the accuracy where $S 1=$ $(0.01,0.01,0.01)$ is close to the center of $\Omega, \mathrm{S} 2=(0.01,0.01,0.99)$ is close to a surface, $\mathrm{S} 3=$ $(0.01,0.99,0.99)$ is closed to an edge and $S 4=(0.99,0.99,0.99)$ is close to a corner.

Fig. 2(a) shows the error convergence in evaluating $V(\boldsymbol{r})$ with varying $a$, where the parameters for the algorithm take $R=1.2 \sqrt{3}, K=3$ and the field point $r=(0.9,0.9,0.9)$. The figure shows that the accuracy is not sensitive to the magnitude of $a$, and $a \approx 50$ is good enough such that the asymptotic error does not influence the numerical approximation.
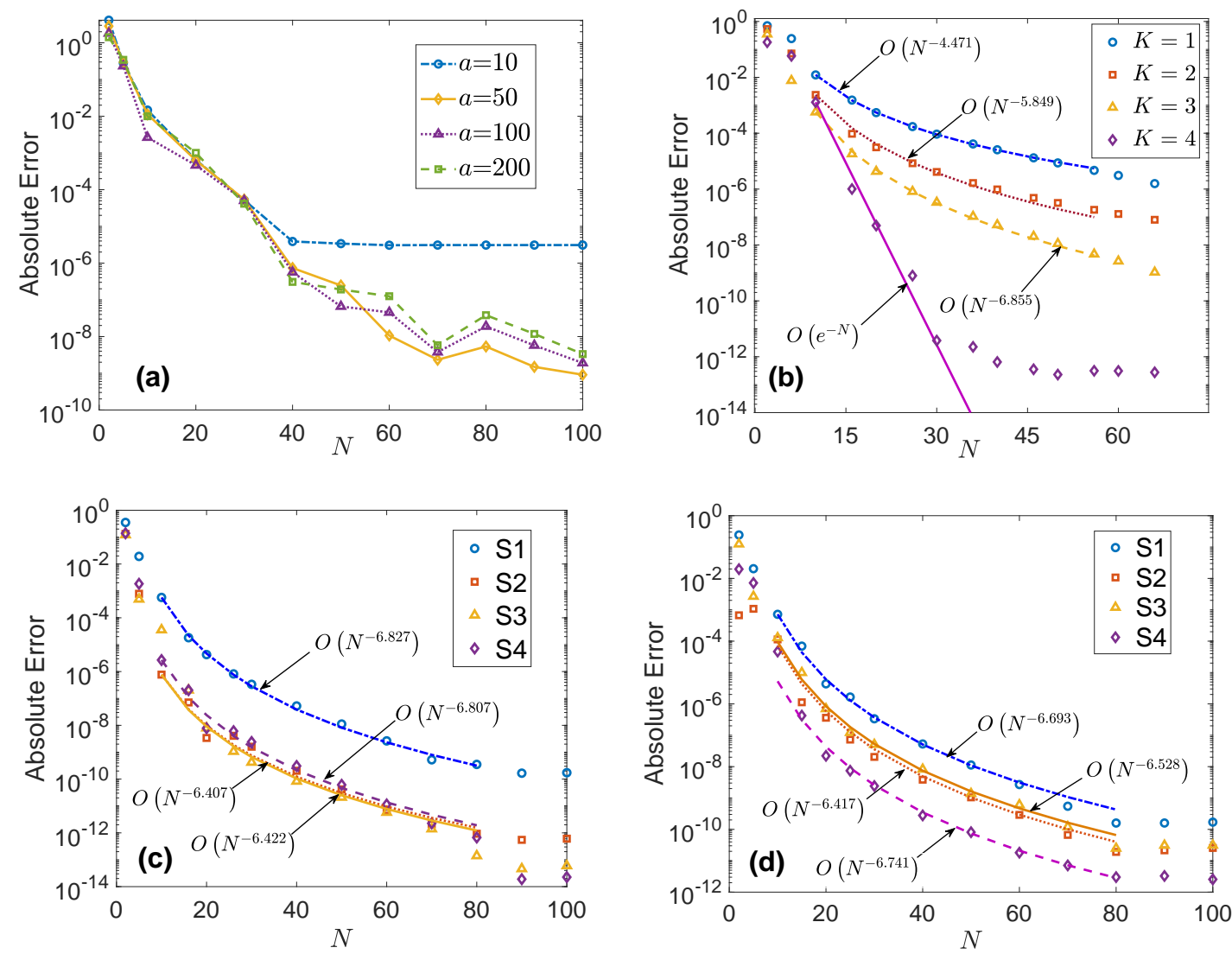

Figure 2: Accuracy and convergence rate for smooth source as function of quadrature point $N$. Panels (a-c) display the accuracy of $V(\boldsymbol{r})$ in the cases of (a) varying the range $a$, (b) varying the Taylor truncation term $K$, and (c) varying the location of the field point; (d) the error of the whole algorithm. Panels (b-d) also show fitted convergence rates. 
Fig. 2(b) takes $R=2 \sqrt{3}, a=50$ and the same field point for different $K$, and the result verifies that the accuracy of the algorithm is about $\mathcal{O}\left(N^{-(K+3)}\right)$ if the source function $f$ exists nonzero partial derivatives of order $K+1$. Fig. 2(c-d) displays the error convergence in evaluating $V(\boldsymbol{r})$ and $u(\boldsymbol{r})$ for the four locations of the field point with the parameters being $R=2 \sqrt{3}, K=3, P=25, a=50$ and $N_{o}=1976$. It is observed that the convergence rate is approximate to $\mathcal{O}\left(N^{-6}\right)$ for all four sites S1-S4.

Example 4.2. We consider the Poisson's equation with a piecewise smooth function $f$ which has a discontinuous derivative,

$$
f(\boldsymbol{r})= \begin{cases}-2\left(z^{2}+2 z\right)\left(x^{2}+y^{2}-2\right), & \text { for } z<0 \\ 0, & \text { for } z \geq 0\end{cases}
$$

with mixed boundary conditions, namely, Neumann boundary on a face at $z=-1$, and Dirichlet boundary on the other five faces. This is more difficult problem due to the non-smoothness of the source and the mixed boundary condition. The same four source points S1-S4 as the previous example are used to test the accuracy.

We test the accuracy of evaluating $V(\boldsymbol{r})$. Fig. 3(a) shows the error convergence for the four field sites with $R=2 \sqrt{3}, K=3, P=25$ and $a=50$. It is observed that the convergence rate remains $\sim \mathcal{O}\left(N^{-6}\right)$ in spite of the nonsmooth source. Fig. 3(b) illustrates errors for the four field sites as function of $N_{0}$. These curves have almost the same tendency, demonstrating that the convergence rate is almost independent of the location of the field point. Fig. 3(c) displays the results as function of $P$ with varying $\lambda=R / \sqrt{3}$ for a given field point $r=\mathrm{S} 1$ and $N_{o}=1976$. Again, the error decreases rapidly with the increase of $P$ and using a larger $\lambda$ will improve the results. Finally, we display the accuracy of the whole algorithm in Fig. 3(d) for this example using the same parameters as panel (a). It is observed that the convergence rate remains to be $\mathcal{O}\left(N^{-6}\right)$, independent of the location of the field point.

To investigate the time performance, Fig. 3(e-f) illustrate the time cost of fast Poisson solver developed in this paper. Each data point has five repeated tests to obtain estimates of the average CPU time. The calculations are performed on an otherwise idle machine (Intel Xeon E5-2680v4, 14 cores, $2.40 \mathrm{GHz}$ ). Fig. 3(e) shows the CPU time for four sets of target points, $W_{1}, W_{2}, W_{3}$ and $W_{4}$, with $R=2 \sqrt{3}, K=3, P=15$ and $a=50$. Each of $W_{1}, W_{2}, W_{3}$ and $W_{4}$ contains 1000 different target points which are closed to the center, a surface, an edge and a corner, respectively. It is observed that the growth rate of CPU time remains linear scaling with increasing $N_{s}$. In panel (f), the CPU time as function of $N_{\text {tar }}$ (the number of target points) is shown for different error levels $\varepsilon_{\infty}=10^{-4}, 10^{-6}, 10^{-8}$ and $10^{-10}$. The maximal absolute error is defined by

$$
\varepsilon_{\infty}:=\frac{\max _{i=1, \cdots, N_{\mathrm{tar}}}\left|u_{\mathrm{HSMA}}^{i}-u_{\mathrm{ref}}^{i}\right|}{\max _{i=1, \cdots, N_{\mathrm{tar}}}\left|u_{\mathrm{ref}}^{i}\right|},
$$



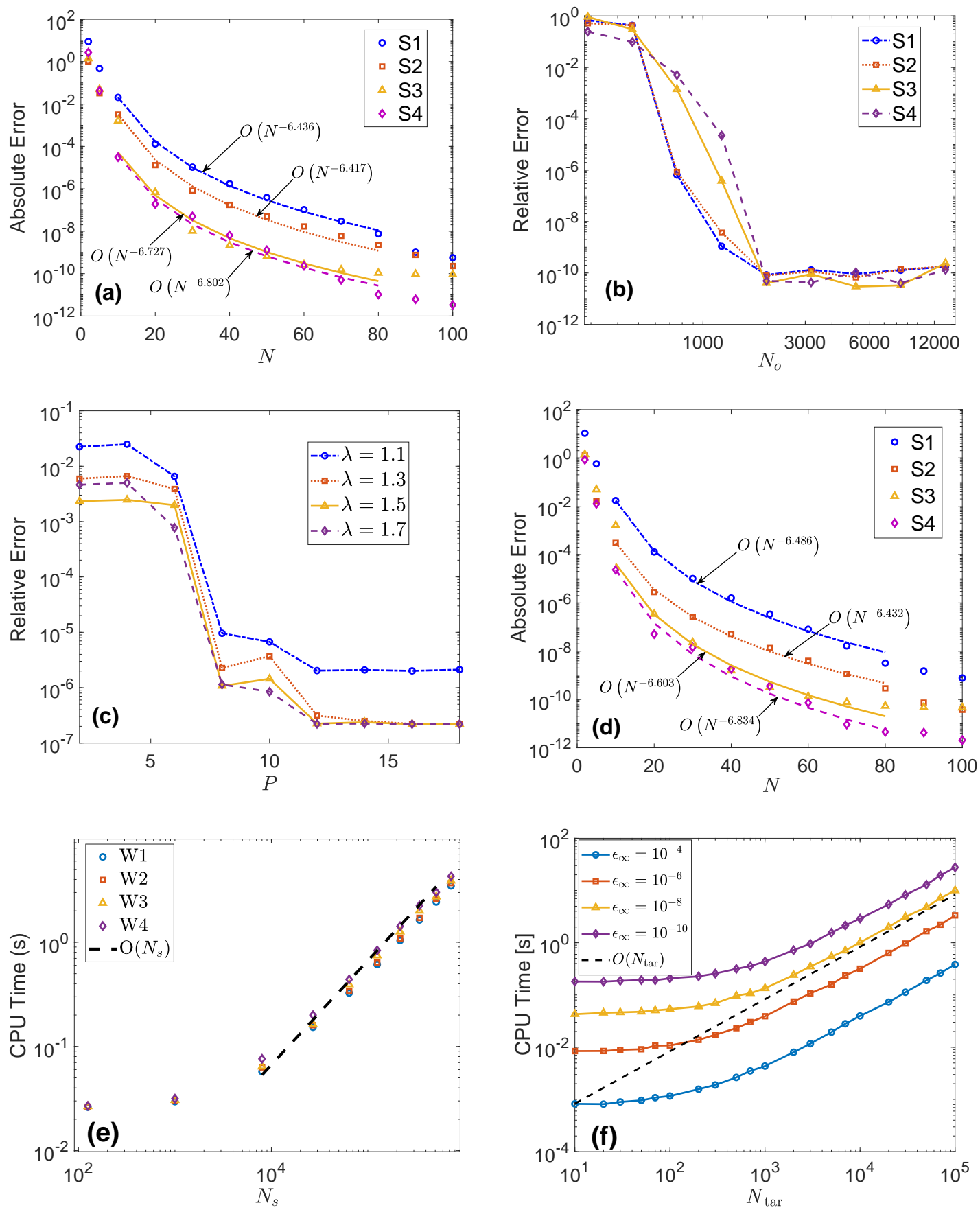

Figure 3: Accuracy, convergence rate and time performance for nonsmooth source. (a) Error in evaluating $V(\mathbf{r})$; (b) error of varying the number $N_{o}$ for $\mathbf{r}=S_{1}$; (c) error with varying the number of the spherical harmonic bases for different $\lambda$ with $N=40$; (d) error of the whole algorithm; (e) CPU time increases with $N_{s}$; (f) CPU time increases with $N_{\text {tar }}$. Panels (a,d) also show the convergence rates by the least square fitting. Black dotted line in panels (e-f) shows the linear scaling. 
Table 1: Algorithm parameters used to determine CPU performance at different error levels.

\begin{tabular}{||l|llll||}
\hline & $\lambda$ & $P$ & $N_{\mathrm{S}}$ & $N_{\mathrm{O}}$ \\
\hline$\varepsilon_{\infty}=10^{-4}$ & 1.5 & 8 & 20 & 756 \\
\hline$\varepsilon_{\infty}=10^{-6}$ & 1.6 & 11 & 40 & 756 \\
\hline$\varepsilon_{\infty}=10^{-8}$ & 1.7 & 15 & 60 & 1222 \\
\hline$\varepsilon_{\infty}=10^{-10}$ & 1.7 & 20 & 80 & 1976 \\
\hline
\end{tabular}

where $u_{\text {HSMA }}^{i}$ is the solution evaluated of the fast Poisson solver developed in this paper and $u_{\text {ref }}^{i}$ is obtain by the same algorithm with parameters enforcing a much higher precision. The target points are all randomly distributed in $\Omega$. The algorithm parameters used to attain these accuracies are listed in Table 1. Unsurprisingly, these curves show $\mathcal{O}\left(N_{\text {tar }}\right)$ scaling. Whereas increasing the accuracy from $\varepsilon_{\infty}=10^{-4}$ to $\varepsilon_{\infty}=10^{-10}$ evidently leads to an increase in CPU time, due to the incorporation of more truncated spherical basis, more quadrature points and larger auxiliary sphere.

Example 4.3. In the third example, we consider the Poisson's equation with the same mixed boundary condition as Example 4.2, but with a discontinuous source $f$ which is 1 for $r \in[-0.5,0.5]^{3}$ and zero elsewhere. Four different source points are used to test the accuracy where $\mathrm{T} 1=\mathrm{S} 1$ is close to the center of $\Omega, \mathrm{T} 2=(0.01,0.01,0.49)$ and $\mathrm{T} 3=(0.01,0.01,0.51)$ are two points close to the discontinuous interface of the source, and $\mathrm{T} 4=\mathrm{S} 4$ is close to a corner point of the central box. We take $N=40, R=2 \sqrt{3}, K=3$, $P=25, a=50$ and $N_{o}=1976$. Fig. 4 shows the accuracy and convergence of evaluating $V(r)$ and $u(\boldsymbol{r})$ respectively in two panels. The spectral convergence for the four sites can be observed.

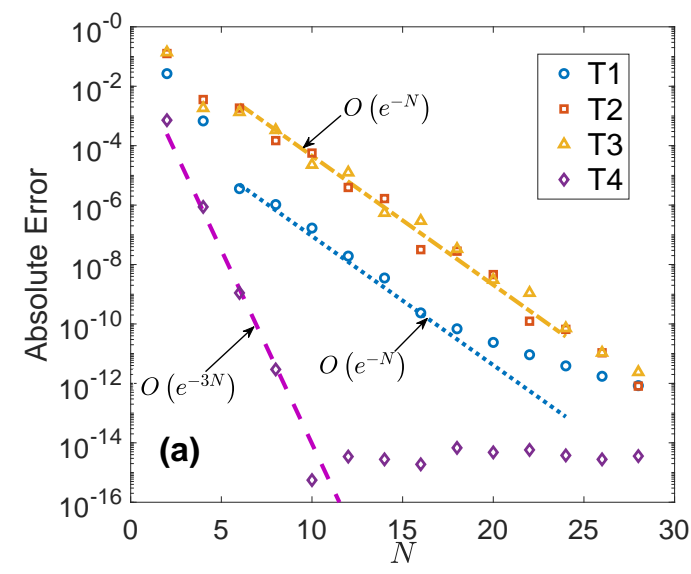

Figure 4: Accuracy and convergence rate for the discontinuous source. (a) Error in evaluating $V(r)$; (b) error of the whole algorithm.

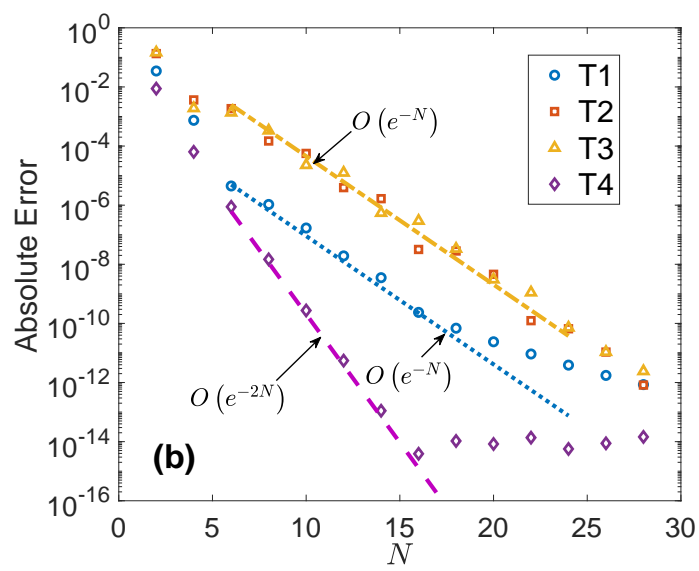




\section{Conclusion}

We have developed an efficient and highly accurate algorithm for solving the Poisson's equation in three dimensional cuboidal domain with mixed boundary conditions, which can reach arbitrary order of accuracy even for a nonsmooth source. By using the ICM and harmonic surface mapping, the algorithm transforms the solution into the superposition of discrete points of Coulomb form and can thus be accelerated by the FMM to achieve linear scaling operation with respect to the number of quadrature points. This method will be promising in many practical applications such as molecular dynamics and electronic structure calculations.

\section{Acknowledgments}

The authors (J. L. and Z. X.) acknowledge the support from the Natural Science Foundation of China (Grant Nos. 12071288 and 21773165), Shanghai Science and Technology Commission (Grant No. 20JC1414100) and the support from the HPC center of Shanghai Jiao Tong University. The author (P. L.) acknowledge the support from the National Science Foundation grant DMS-1816740.

\section{References}

[1] R. W. Hockney, J. W. Eastwood, Computer Simulation Using Particles, Taylor \& Francis, 1988.

[2] D. Frenkel, B. Smit, Understanding Molecular Simulation: From Algorithms to Applications, Elsevier, 2001.

[3] J. P. Verboncoeur, Particle simulation of plasmas: Review and advances, Plasma Phys. Control. Fusion 47 (5A) (2005) A231.

[4] J. J. Monaghan, J. C. Lattanzio, A refined particle method for astrophysical problems, Astron. Astrophys. 149 (1985) 135-143.

[5] B. Zhang, J. DeBuhr, D. Niedzielski, S. Mayolo, B. Lu, T. Sterling, DASHMM accelerated adaptive fast multipole Poisson-Boltzmann solver on distributed memory architecture, Commun. Comput. Phys. 25 (2019) 1235-1258.

[6] X. Cao, H. Huang, An adaptive conservative finite volume method for Poisson-NernstPlanck equations on a moving mesh, Commun. Comput. Phys. 26 (2019) 389-412.

[7] H. Liu, W. Maimaitiyiming, Unconditional positivity-preserving and energy stable schemes for a reduced Poisson-Nernst-Planck system, Commun. Comput. Phys. 27 (2020) 1505-1529.

[8] I. Kwon, D. Kwak, Discontinuous bubble immersed finite element method for PoissonBoltzmann equation, Commun. Comput. Phys. 25 (2019) 928-946.

[9] V. Thomée, From finite differences to finite elements: A short history of numerical analysis of partial differential equations, J. Comput. Appl. Math. 128 (1-2) (2001) 1-54.

[10] N. Nishimura, Fast multipole accelerated boundary integral equation methods, Appl. Mech. Rev. 55 (4) (2002) 299-324.

[11] W. Ying, C. S. Henriquez, A kernel-free boundary integral method for elliptic boundary value problems, J. Comput. Phys. 227 (2) (2007) 1046-1074. 
[12] G. C. Hsiao, O. Steinbach, W. L. Wendland, Boundary Element Methods: Foundation and Error Analysis, Wiley Online Library, 2017, Ch. 12, pp. 339-373.

[13] L. Greengard, V. Rokhlin, A new version of the fast multipole method for the Laplace equation in three dimensions, Acta Numer. 6 (1997) 229-269.

[14] L. Ying, G. Biros, D. Zorin, A kernel-independent adaptive fast multipole algorithm in two and three dimensions, J. Comput. Phys. 196 (2) (2004) 591-626.

[15] A. Gholami, D. Malhotra, H. Sundar, G. Biros, FFT, FMM, or multigrid? A comparative study of state-of-the-art Poisson solvers for uniform and nonuniform grids in the unit cube, SIAM J. Sci. Comput. 38 (3) (2016) 280-306.

[16] J. Shen, Efficient spectral-Galerkin method I. Direct solvers of second- and fourth-order equations using Legendre polynomials, SIAM J. Sci. Comput. 15 (6) (1994) 1489-1505.

[17] J. Shen, Efficient spectral-Galerkin method II. Direct solvers of second- and fourth-order equations using Chebyshev polynomials, SIAM J. Sci. Comput. 16 (1) (1995) 74-87.

[18] E. Braverman, M. Israeli, A. Averbuch, L. Vozovoi, A fast 3D Poisson solver of arbitrary order accuracy, J. Comput. Phys. 144 (1) (1998) 109-136.

[19] A. Averbuch, M. Israeli, L. Vozovoi, A fast poisson solver of arbitrary order accuracy in rectangular regions, SIAM J. Sci. Comput. 19 (3) (1998) 933-952.

[20] F. Ethridge, L. Greengard, A new fast-multipole accelerated Poisson solver in two dimensions, SIAM J. Sci. Comput. 23 (3) (2001) 741-760.

[21] N. A. Gumerov, R. Duraiswami, A method to compute periodic sums, J. Comput. Phys. 272 (2014) 307-326.

[22] L. Greengard, J. Y. Lee, A direct adaptive Poisson solver of arbitrary order accuracy, J. Comput. Phys. 125 (2) (1996) 415-424.

[23] C. R. Anderson, A method of local corrections for computing the velocity field due to a distribution of vortex blobs, J. Comput. Phys. 62 (1) (1986) 111-123.

[24] P. McCorquodale, P. Colella, G. Balls, S. Baden, A local corrections algorithm for solving poisson's equation in three dimensions, Comm. Appl. Math. Comput. Sci. 2 (1) (2007) 57-81.

[25] M. M. Hejlesen, J. T. Rasmussen, P. Chatelain, J. H. Walther, A high order solver for the unbounded Poisson equation, J. Comput. Phys. 252 (2013) 458-467.

[26] F. Vico, L. Greengard, M. Ferrando, Fast convolution with free-space Green's functions, J. Comput. Phys. 323 (2016) 191-203.

[27] Q. Zhao, J. Liang, Z. Xu, Harmonic surface mapping algorithm for fast electrostatic sums, J. Chem. Phys. 149 (8) (2018) 084111.

[28] J. Liang, J. Yuan, E. Luijten, Z. Xu, Harmonic surface mapping algorithm for molecular dynamics simulations of particle systems with planar dielectric interfaces, J. Chem. Phys. 152 (13) (2020) 134109.

[29] J. D. Jackson, Classical Electrodynamics (3nd Edition), John Wiley \& Sons, New York, 2001.

[30] W. Cai, S. Deng, D. Jacobs, Extending the fast multipole method to charges inside or outside a dielectric sphere, J. Comput. Phys. 223 (2007) 846-864.

[31] Q. Zhao, P. Liu, Z. Xu, A fast method for evaluating Green's function in irregular domains with application to charge interaction in a nanopore, Commun. Comput. Phys. 24 (4) (2018) 1241-1258.

[32] T. A. Darden, D. M. York, L. G. Pedersen, Particle mesh Ewald: An N $\log (N)$ method for Ewald sums in large systems, J. Chem. Phys. 98 (1993) 10089-10092.

[33] U. Essmann, L. Perera, M. L. Berkowitz, T. Darden, H. Lee, L. Pedersen, A smooth particle mesh Ewald method, J. Chem. Phys. 103 (1995) 8577-8593.

[34] J. Shimada, H. Kaneko, T. Takada, Efficient calculations of Coulombic interactions in 
biomolecular simulations with periodic boundary conditions, J. Comput. Chem. 14 (1993) 867-878.

[35] B. A. Luty, M. E. Davis, I. G. Tironi, W. F. Van Gunsteren, A comparison of particleparticle, particle-mesh and Ewald methods for calculating electrostatic interactions in periodic molecular systems, Mol. Simul. 14 (1994) 11-20.

[36] J. J. Thomson, On the structure of the atom: An investigation of the stability and periods of oscillation of a number of corpuscles arranged at equal intervals around the circumference of a circle; with application of the results to the theory of atomic structure, Phil. Mag. Ser. 6 7 (39) (1904) 237-265.

[37] J. Fliege, U. Maier, The distribution of points on the sphere and corresponding cubature formulae, IMA J. Numer. Anal. 19 (2) (1999) 317-334(18).

[38] M. Abramowitz, I. Stegun, D. A. Mcquarrie, Handbook of Mathematical Functions, 1970.

[39] J. H. Hannay, J. F. Nye, Fibonacci numerical integration on a sphere, J. Phys. A: Math. Gen. 37 (48) (2004) 11591.

[40] M. M. Chawla, M. K. Jain, Asymptotic error estimates for the Gauss quadrature formula, Math. Comput. 22 (101) (1968) 91-97.

[41] J. D. Murray, Asymptotic Analysis, Springer-Verlag, Berlin, 1984.

[42] W. Fong, E. Darve, The black-box fast multipole method, J. Comput. Phys. 228 (23) (2009) $8712-8725$. 